\title{
ON POLYNOMIAL EQUATIONS IN BANACH SPACE, PERTURBATION TECHNIQUES AND APPLICATIONS
}

\section{IOANNIS K. ARGYROS}

\author{
Department of Mathematics \\ The University of Iowe \\ Iowa City, Iowa $5224 a$
}

(Received November 21, 1985 and in revised form May 23 1986)

ABSTRACT. We use perturbation techniques to solve the polynomial equation in Banach space. Our techniques provide more accurate information on the location of solutions and yield existence and uniqueness in cases not covered before. An example is given to justify our method.

KEY WORDS AND PHRASES. Perturbation, contraction. 1980 AMS SUBJECT CLASSIFICATION CODE(S). 46B15,65

\section{INTRODUCTION.}

In this paper we use perturbation techniques to find solutions of the abstract polynomial equation of degree $k$,

$$
x=P_{k}(x)=M_{k} x^{k}+M_{k-1} x^{k-1}+\cdots+M_{1} x+M_{0}
$$

in a Banach space $X$ over the field $F$ of real or complex numbers.

Obviously (1.1) is a natural generalization of the scalar polynomial equation of the first kind to the more abstract setting of a Banach space.

The case $k=2$ has been examined in [1], [7], [8]. Here we investigate the case $k \geq 2$. The principal new idea in this paper is the introduction of an equation similar to (I.I),

$$
z=F_{k}(z)=N_{k} z^{k}+N_{k-1} z^{k-1}+\cdots+N_{2} z^{2}+N_{1} z+M_{0} .
$$

The 'results are then obtained under suitable choices of the $N_{p}$ 's, $p=1,2, \cdots, k$. our method is a generalization of the one's discussed [8], [9] by L. B. Rall, namely, the method of successive substitutions and Newton's method. It always provides a more accurate information on the location of the solutions and it also yields existence and uniqueness results for (I.I) in the cases not covered before. For $\mathbf{z}=0$ and $A_{2}=M_{2}-I$ our results coincide with theorems in [8], [9], but even then we are able to provide more accurate information on the location of the solutions. In order to justify this, in Part 2 of our paper we compare our results with the results in [8], [9], [10] using as an example a special case of (1.1), namely the famous Chandrasekhar's equation 


$$
x i s ;=I+\lambda z i=1 \int_{-} \frac{s}{s+i} x(t) d i
$$

for $X=C[0,1 j$ and $0 \leq \lambda \leq 0.5$. Stronger results for an even more general form of (1.3) have already been obtained in [3j, [6] ana elsewhere. In tris paper we just us (1.3) as an example to justify our metnoc.

2. BASIC CONCEPTS AND THEOREME.

DEFINITION $I$. Denote by $L(X, Y)$ the linear space over the field $F$ of the linear operators from a linear space $X$ into a linear space $Y$. For $k=2,3, \cdots$ a linear operator from $X$ into the space $L\left(X^{k-1}, Y\right)$ of $(k-1)$-linear operators from $X$ into $Y$ is called $\underline{k-l i n e a r}$ operator from $X$ into $Y$. For example, if a k-linear operator $M_{k}$ from $X$ into $Y$ and $k$ points $x_{1}, x_{2}, \cdots, x_{k} \in X$ are given, then

$$
z=M_{k} x_{1} x_{2} \cdots x_{k}
$$

will be a point of $Y$, the convention being that $M_{k}$ operates on $x_{1}$, the (k-1)linear operator $\mathrm{M}_{\mathrm{k}} \mathrm{x}_{1}$ operates on $\mathrm{x}_{2}$, and so on. The order of operation is important. Finally, denote $L(X, Y)$ by $L(X)$ if $X=Y$.

NOTATION 1. Given a $k$-linear operator $M_{K}$ from $X$ into $Y$ and a permutation $i=\left(i_{1}, i_{2}, \cdots, i_{k}\right)$ of the integers $1,2, \cdots, k$, the notation $M_{k}(i)$ can be used for the $k$-linear operator from $X$ into $Y$ such that

for all $x_{1}, x_{2}, \cdots, x_{k} \in X$.

$$
M_{k} \text { (i) } x_{1} x_{2} \cdots x_{k}=M_{k} x_{i_{1}} x_{i_{2}} \cdots x_{i_{k}}
$$

Thus, there are $k$ ! $k$-linear operators $M_{k}(i)$ associated with a given $k$-linear operator $\mathrm{M}_{\mathrm{k}}$.

DEFINITION 2. A k-linear operator $M_{k}$ from $X$ into $Y$ is said to be symmetric if

$$
M_{k}=M_{k}(i)
$$

for all $i \in R_{k}$, where $R_{k}$ denotes the set of all permutations of the integers $1,2, \cdots, \mathrm{k}$. The symetric $\mathrm{k}$-linear operator

is called the mean of $\mathrm{M}_{\mathrm{K}}$.

$$
\bar{M}_{k}=\frac{1}{k !} \sum_{i \in R_{k}} M_{k}(i)
$$

NOTATION 2. The notation

$$
M_{k} x^{p}=M_{k} \underset{x x \cdot \cdots x}{p}
$$

$p \leq k, M_{k} \in L\left(X^{k}, Y\right)$, for the result of applying $M_{k}$ to $x \in X$ p-times will be used. If $p<k$, then $M_{k} x^{p}$ will represent a $(k-p)$-linear operator from $X$ into Y. For $p=k$, note that

$$
M_{k} x^{k}=\bar{M}_{k} x^{k}=M_{k}(i) x^{k}
$$

for all $i \in R_{k}$, $x \in X$. It follows from (2.1) that the multilinear operators $M_{2}, \cdots, M_{k}$ in (1.I) may be assumed to be symmetric without loss of generality, since each $M_{i}$ in (I.I) may be replaced by $\bar{M}_{i}, i=2,3, \cdots, k$, without changing the value 
of $\xi_{k}\left(x_{i}\right.$. uniess tne contrary is expicitly state $\dot{s}$, the multilinear operators $y_{i}$, $\dot{\Sigma}=\varepsilon, \bar{j}, \cdots, k$ will be assumed to be symmetric.

Assume from now or that $\mathrm{X}, \mathrm{V}$ are Banach spaces.

DEFINITION $j$. A. Iinear operator $I$ from $y$ into $Y$ is said to be bounded is

$$
\|\mathrm{L}\|=\sup _{\|\mathrm{x}\|=2}\|\mathrm{~L} x\|_{i}
$$

is finite. The quantity $\|\mathrm{L}\|$ is called the bound (or norm) of $i$.

DEFINITION 4. For $\mathrm{k} \geq 2$, \& $\mathrm{k}$-linear operator $\mathrm{N}_{\mathrm{k}}$ from $\mathrm{X}$ into $\mathrm{Y}$ is said to be bounded if it is a bounded linear operator from $X^{K}$ into $L\left(X^{k-I}, Y\right)$, the Banach space of bounded (k-I)-linear operators from $X$ into $Y$. The bound (or norm) $\mid \mathbb{M}_{K} \|$ of $\mathrm{N}_{\mathrm{k}}$ is defined by (2.2), with $\mathrm{M}_{\mathrm{k}}$ being considered to be an element of $\mathrm{L}\left(\mathrm{X}, \mathrm{L}\left(\mathrm{X}^{\mathrm{k}-1}, \mathrm{Y}\right)\right)$.

NOTATION 3. The space of bounded k-linear operators from $X$ into $Y$ will be denoted henceforth by $L\left(X^{k}, Y\right)$. Note that by Definitions (3) and (4) if $M_{k} \in I\left(X^{k}, Y\right)$ and $\mathrm{p} \leq \mathrm{k}$ then

$$
\left\|\mathrm{M}_{\mathrm{k}} \mathrm{x}^{\mathrm{p}}\right\| \leq\left\|\mathrm{M}_{\mathrm{k}}\right\| \quad\|\mathrm{x}\|^{\mathrm{p}} \text {. }
$$

DEFINITION 5. An abstract polynomial operator $P_{k}$ from $X$ into $Y$ of degree k defined by

$$
P_{k}(x)=M_{k} x^{k}+M_{k-1} x^{k-1}+\cdots+M_{2} x^{2}+M_{1} x+M_{0},
$$

is said to be bounded if its coefficients $M_{i}, i=1,2, \cdots, k$ are bounded multilinear operators from $X$ into $Y$. From now on we assume $P_{k}$ is bounded.

DEFINITION 6. Let $\mathrm{z}$ be fixed in $\mathrm{X}$ and define the polynomial $\mathrm{q}_{\mathrm{k}}$ of degree $\mathrm{k}$ on $\mathbb{R}^{+}$by

$$
q_{k}(r)=\left\|P_{k}(z)-z\right\|+\left\|M_{1}\right\|\left[\frac{(r+\|z\|)-\|z\|}{(r+\|z\|)-\|z\|}\right] r+\cdots+\left\|M_{k}\right\|\left[\frac{(r+\|z\|)^{k}-\|z\|^{k}}{(r+\|z\|)-\|z\|}\right] r .
$$

Note that by Descartes rule of signs [5] the equation $q(r)=q_{k}(r)-r=0$ has two positive solutions $s_{1} \leq s_{2}$ or none.

THEOREM 1. Assume that $q(r)$ has two positive solutions $s_{1}<s_{2}$ such that $q^{\prime}(r)<1, \quad r \in\left(s_{1}, s_{2}\right)$. Then $P_{k}$ has a unique fixed point in the ball $\bar{U}(z, r)=$ $\{x \in X \mid\|x-z\| \leq r\}$, where $r \in\left(s_{1}^{\prime}, s_{2}^{\prime}\right) \subset\left(s_{1}, s_{2}\right)$.

PROOF. Claim $1 . \mathrm{P}_{\mathrm{k}}$ maps $\overline{\mathrm{U}}(\mathrm{z}, \mathrm{r})$ into $\overline{\mathrm{U}}(\mathrm{z}, \mathrm{r})$.

$$
\begin{aligned}
\left\|P_{k}(x)-z\right\| & =\left\|P_{k}(x)-P_{k}(z)+P_{k}(z)-z\right\| \leq\left\|P_{k}(x)-P_{k}(z)\right\|+\left\|P_{k}(z)-z\right\| \\
& \leq\left\|M_{I}(x-z)+\cdots+M_{k}(x-z) x^{k-1}+M_{k} x^{k-2}(x-z) z+\cdots+M_{k} z^{k-1}(x-z)\right\| \\
& \leq\left[\left\|M_{I}\right\|+\left\|M_{2}\right\|(r+\|z\|)+\cdots+\left\|M_{k}\right\|\left((r+\|z\|)^{k-1}+(r+\|z\|)^{k-2}\|z\|+\cdots+\|z\|^{k-1}\right] r\right. \\
& +\left\|P_{k}(z)-z\right\| \leq r
\end{aligned}
$$

or

$$
q(r) \leq 0 \text { which is true by hypothesis. }
$$

(Note that claim 1 is true even if $s_{1}=s_{2}$ and $r \in\left[s_{1}, s_{2}\right]$ ).

Claim 2. $P_{k}$ is a contraction operator on $\bar{U}(z, r)$. IF $x_{1}, x_{2} \in \bar{U}(z, r)$, then as in claim 1, 
bui

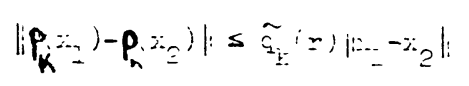

$$
\tilde{q}_{k:}(r)<\sum \text { by hypothesis. }
$$

The result now follows from the contraction mappine principle.

DEFINITION $\tau$. Define the polynomiai $\tilde{q}_{k}(r)$ of degree $x$ on $\mathbb{I}_{i}^{+}$by

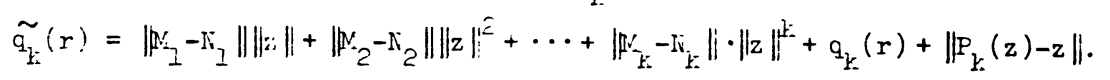

Note that

$$
\begin{aligned}
\left\|P_{k}(z)-z\right\| & =\left\|M_{0}+M_{1} z+M_{2} z^{2}+\cdots+M_{k} z^{k}-z\right\| \\
& =\left\|M_{0}-M_{0}+M_{1} z-N_{1} z+\cdots+M_{k} z^{k}-N_{k} z^{k}+\left(F_{k}(z)-z\right)\right\| \\
& \leq\left\|M_{1}-N_{1}\right\| \cdot\|z\|+\left\|M_{2}-N_{2}\right\| \cdot\|z\|^{2}+\cdots+\left\|M_{k}-N_{k}\right\| \cdot\|z\|^{k}
\end{aligned}
$$

if $\mathrm{z}$ is a fixed point of (1.2).

The proof of the following theorem follows from Theorem $I$ and the above observation.

THEOREM 2. Suppose that there exists a solution $z$ satisfying (1.2) and that $\tilde{q}(r)=\tilde{q}_{k}(r)-r$ has two positive solutions $s_{1}<s_{2}$. Then $P_{k}$ has a unique fixed point in $\bar{U}(z, r)$, where $r \in\left(s_{1}^{\prime}, s_{2}^{\prime}\right) \subset\left(s_{1}, s_{2}\right)$.

\section{APPLICATIONS.}

From now on we assume that $k=2$ and $M_{1}=0$. Then Theorem 2 becomes

THEOREM 3. Consider the equation

$$
z=M_{0}+N_{2} z^{2}
$$

Suppose that there exists a solution $z$ satisfying (3.1) and

$$
\|z\|<\left[2 \sqrt{\left\|\mathrm{M}_{2}\right\|}\left(\sqrt{\left\|\mathrm{M}_{2}-\mathrm{N}_{2}\right\|}+\sqrt{\left\|\mathrm{M}_{2}\right\|}\right)\right]^{-1} \text {. }
$$

(I) Then the equation

$$
x=M_{0}+M_{2} x^{2}
$$

has a unique solution $x \in U(z, a)$, where

$$
a=\frac{1}{2\left\|m_{2}\right\|}-\|z\| \text {. }
$$

(II) Moreover, $x \in \bar{U}(z, b)$ where

$$
\mathrm{b}=\left\{1-2\left\|\mathrm{M}_{2}\right\| \cdot\|z\|-\left[\left(2\left\|\mathrm{M}_{2}\right\| \cdot\|z\|-1\right)^{2}-4\left\|\mathrm{M}_{2}-\mathrm{N}_{2}\right\|\left\|\mathrm{M}_{2}\right\| \cdot\|z\|^{2}\right]^{1 / 2}\right\}\left(2\left\|\mathrm{M}_{2}\right\|\right)^{-1} .
$$

In practice, an exact solution of the auxiliary equation (3.1) can seldom be obtained. The following theorem, whose proof is similar to that of Theorem 2, guarantees that the original equation (3.2) has a solution even when we can only find an approximate solution of (3.1).

THEOREM 4. Let $z$ be fixed in $X$ and set

$$
\begin{aligned}
& a=\frac{1}{2\left\|w_{2}\right\|}-\|z\| \\
& \epsilon=\left\|\mathbb{N}_{2} z^{2}+M_{0}-z\right\| \cdot\|z\|^{2} \\
& b=a-\left[a^{2}-\frac{\left\|M_{2}-N_{2}\right\|\|z\|^{2}+\epsilon\|z\|^{2}}{\left\|M_{2}\right\|}\right]^{1 / 2} .
\end{aligned}
$$


s.esums the:

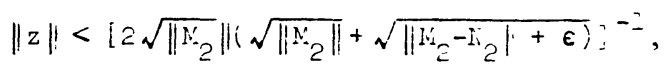

tner.

(I) equation (3.2) has a unique solution in $\mathrm{Uiz}, \mathrm{a}$ );

(II) this soiution actually lies in $\bar{U}(z, b)$.

PROOI. Let us define the operator on $X \quad b y$

$$
\mathrm{T}(\mathrm{x})=\mathrm{N}_{0}+\mathrm{N}_{2} \mathrm{x}^{2} \text {. }
$$

Claim: 1. T maps $\bar{U}(z, r)$ into $\bar{U}(z, r)$ for $r \in[b, a)$. If $x \in \bar{U}(z, r)$ then

$$
\begin{aligned}
T(x)-z & =M_{2} x^{2}+M_{0}-z \\
& =\left(N_{2}-N_{2}\right)(x-z+z)^{2}+N_{2}(x-z)^{2}+2 N_{2} z(x-z)+\left(N_{2} z^{2}+M_{0}-z\right) .
\end{aligned}
$$

Now, $\|T(x)-z\| \leq r$ if

$$
\left(\left\|\mathrm{M}_{2}-\mathrm{N}_{2}\right\|+\left\|\mathrm{N}_{2}\right\|\right) r^{2}+\left(2\|\mathrm{z}\| \mathrm{m}_{2}-\mathrm{N}_{2}\|+2\| \mathrm{N}_{2}\|\cdot\| \mathrm{z} \|-\mathrm{I}\right) \mathrm{r}+\left\|\mathrm{M}_{2}-\mathrm{N}_{2}\right\| \cdot\|\mathrm{z}\|^{2}+\epsilon\|z\|^{-2} \leq 0
$$

which is true for $r \in[a, b)$.

Claim 2. $T$ is a contraction operator on $\bar{U}(z, r)$. If $w, v \in \bar{U}(z, r)$ then

$$
\begin{aligned}
\|T(w)-T(v)\| & =\left\|M_{2} w^{2}-M_{2} v^{2}\right\| \\
& =\left\|M_{2}(w-z+v-z+2 z)(w-v)\right\| ! \\
& \leq 2(r+w z \|)\|w-v\|\left\|M_{2}\right\| i
\end{aligned}
$$

So $T$ is a contraction on $\bar{U}(z, r)$ for $0<r<a$.

Because Theorem 4 relies on the contraction mapping principle, it actually provides an iteration procedure for solving (3.2), namely, set

$$
\begin{aligned}
& x_{0}=z \quad \text { and } \\
& x_{n+1}=M_{0}+M_{2} x_{n}^{2}, \quad n=1,2, \cdots .
\end{aligned}
$$

REMARK 1. The iteration

$$
x_{n+1}=M_{0}+M_{2} x_{n}^{2}, \quad n=1,2, \cdots
$$

converges for any $x_{0} \in \bar{U}(z, b)$ to the solution $x$ of (3.2) at the rate of a geometric progression with quotient

$$
q=1-\left[\left(2\left\|\mathbb{M}_{2}\right\| \cdot\|z\|-1\right)^{2}-4 \mathbb{M}_{2}-N_{2}\|\| M_{2}\|\cdot\| z \|^{2}\right]^{1 / 2} \text {. }
$$

PROOF. By Theorem 3 we have

$$
\begin{aligned}
\mathrm{q} & =2(\mathrm{~b}+\|\mathrm{z}\|)\left\|\mathrm{M}_{2}\right\| \\
& =1-\left[\left(2\left\|\mathrm{M}_{2}\right\| \cdot\|z\|-1\right)^{2}-4\left\|\mathrm{M}_{2}\right\| \cdot\left\|\mathrm{M}_{2}-\mathrm{N}_{2}\right\| \cdot\|z\|^{2}\right]^{1 / 2} .
\end{aligned}
$$

COROLLARY 1. Under the hypotheses of Theorem 3, the solution $x$ obtained in Theorem 3 satisfies

PROOF. By Theorem 3,

$$
\|x\|<\frac{1}{2\|M\|}
$$

$$
\|x-z\|<a,
$$


so tinat

$$
\begin{aligned}
& \|x\| \leq\|z\|+c_{1}, \\
& \|x\|<\frac{\vdots}{2 \| N_{2} \mid} .
\end{aligned}
$$

COROLLARY 2. For any ${ }^{\mathrm{N}_{\mathrm{C}}} \in \mathrm{X}$ such that $4\left\|\mathrm{M}_{2}\right\| \cdot\left\|\mathrm{M}_{0}\right\|<1$,

(I) equation ( 3.2$)$ has a unique solution $x \in U\left(N_{1}, a\right)$, where

$$
a=\frac{1-2\left\|M_{2}\right\|\left\|M_{0}\right\|}{2\left\|M_{2}\right\|}
$$

(II) moreover, $x \in \bar{U}\left(M_{0}, b\right)$ where

$$
b=\frac{1-2\left\|M_{2}\right\| \cdot\left\|M_{0}\right\|-\sqrt{1-4\left\|M_{2}\right\| \cdot\left\|M_{0}\right\|}}{2\left\|M_{2}\right\|} .
$$

PROOF. Apply Theorem 3 with $M_{2}=0$ and $z=N_{1_{0}}$.

We now state Rall's theorem for comparison. The proof can be found in [8], [9]. THEOREM 5. If $4\left\|M_{2}\right\| \cdot\left\|M_{0}\right\|<I$ then

(I) equation (3.2) has a solution $x \in X$ satisfying

$$
\|x\| \leq \frac{1-\sqrt{1-4\left\|M_{2}\right\|\left\|M_{0}\right\|}}{2\left\|M_{2}\right\|}
$$

(II) moreover, $x$ is unique in $U(x, R)$, where

$$
R=\frac{\sqrt{1-4\left\|M_{2}\right\|\left\|M_{0}\right\|}}{2\left\|M_{2}\right\|} .
$$

PROPOSITION 1. Assume:

(I) the hypotheses of Theorems 3, 5 are satisfied;

(II) $\left(\left\|M_{2}\right\|-\| M_{2}-N_{2} i\right)\|z\|^{2}-\|z\|+\left\|M_{0}\right\|>0$.

Then Theorem 3 provides a sharper estimate on $\|x\|$ than Theorem 5 .

PROOF. By Theorem 3,

$$
\|x-z\| \leq b \quad \text { so } \quad\|x\| \leq b+\|z\| \text {. }
$$

By Theorem 5,

$$
x \leq \frac{1-\sqrt{1-4\left\|M_{2}\right\|\left\|M_{0}\right\|}}{2\left\|M_{2}\right\|}
$$

so it is enough to show

$$
\frac{1-\left[\left(2\left\|M_{2}\right\| \cdot\left\|M_{0}\right\|-1\right)^{2}-4\left\|M_{2}\right\|\left\|M_{2}-N_{2}\right\|\|z\|^{2}\right]^{1 / 2}}{2\left\|M_{2}\right\|}<\frac{1-\sqrt{1-4\left\|M_{2}\right\|\left\|M_{0}\right\|}}{2\left\|M_{2}\right\|}
$$

or

$$
\left(\left\|M_{2}\right\|-\left\|M_{2}-N_{2}\right\|\right)\|z\|^{2}-\|z\|+\|y\|>0
$$

and the result follows from (II).

REMARK 2. If the evaluation of $\left\|M_{2}-\mathrm{N}_{2}\right\|$ in Theorem 3 is difficult, then

(a) we can look for a 2 such that: 


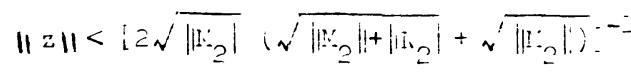

$$
\begin{aligned}
& \leq\left[2 \sqrt { \| M _ { 2 } \| } \left(\sqrt{\left\|M_{2}-N_{2}\right\|}+\sqrt{\left\|N_{2}\right\|} j^{-I}\right.\right.
\end{aligned}
$$

and start the iteration witr $y_{C}=z$;

(b; we can apply tne theorer in the ball $\bar{U}\left(z, \dot{a}^{\prime} ;\right.$. where $\mathrm{b} \leq \mathrm{c}^{\prime}<\mathrm{a}$ and

$$
a^{\prime}=\frac{I-2\left\|M_{2}\right\| \cdot\|z\|-\left[\left(2\left\|M_{2}\right\| \cdot\|z\|-I\right)^{2}-4\left\|M_{2}\right\|+\left\|N_{2}\right\|\right)\|z\|_{2}^{2} I / z}{2\left\|N_{2}\right\|},
$$

provided that the quantity under the radical is nonnegative. Also note that sice $\mathrm{b} \leq \mathrm{a}^{\prime}<\mathrm{a}$, we have

$$
\bar{U}(z, b) \subset \bar{U}\left(z, a^{\prime}\right) \subset U(z, a)
$$

EXAMPLE 1. For the equation of Chandrasekhar,

$$
x(s)=I+\lambda x(s) \int_{0}^{1} \frac{s}{s+t} x(t) d t
$$

we have $\mathrm{X}=\mathrm{C}[0,1]$ with the sup-norm. The operator $Q: X \rightarrow X$ defined by

$$
Q(x)=x(s) \int_{0}^{1} \frac{s}{s+t} x(t) d t
$$

is quadratic since the symmetric bilinear operator $M_{2}: X \times X \rightarrow X$ defined by

$$
M_{2}(x, y)=\frac{1}{2}\left[x(s) \int_{0}^{1} \frac{s}{s+t} y(t) d t+y(s) \int_{0}^{1} \frac{s}{s+t} x(t) d t\right]
$$

satisfies

$$
M_{2}(x, x)=Q(x) \text { for all } x \in X \text {. }
$$

We will prove that the norm $\left\|\mathrm{M}_{2}\right\|=$ In 2. Now

$$
\|Q\|=\max _{s} \int_{0}^{l}\left|\frac{s}{s+t}\right| d t=\ln 2
$$

and since always

we obtain

$$
\|Q\| \leq\left\|M_{2}\right\|
$$

$$
\text { In } 2 \leq\left\|\mathrm{M}_{2}\right\| \text {. }
$$

The proof will be completed if we prove that

$$
\left\|M_{2}\right\| \leq \ln 2 \text {. }
$$

But by the definition of $\mathrm{M}_{2}$,

so

$$
\left\|M_{2}\right\| \leq \frac{1}{2} \max _{s} 2 \int_{0}^{l}\left|\frac{s}{s+t}\right| d t=\ln 2
$$

$$
\left\|\mathrm{M}_{2}\right\|=\ln 2 \text {. }
$$

We now apply Theorem 5 and Corollary 2 to (3.3) with $B=\lambda N_{2}$. According to Theorem 5 , equation (3.3) has a unique solution in $U(x, R)$, where

$$
R=\frac{\sqrt{1-4 \lambda \ln 2}}{2 \lambda \ln 2}
$$

provided that $1-4 \lambda$ In $2>0$, i.e., $\lambda<.36067 \cdots$. According to Corollary 2 , 
(I) equatior ( $\left(.3\right.$, has a unique sollitior ar $U\left(x, E_{1}\right.$, where

$$
i=\frac{I-2 \lambda \text { in } z}{2 \lambda \text { Ir. } \bar{c}}
$$

(II) moreover, $\mathrm{x} \in \overline{\mathrm{U}}(I, \mathrm{a})$, wher

$$
a=\frac{1-2 \lambda \ln 2-\sqrt{1-4 \lambda \ln 2}}{2 \lambda \ln 2},
$$

provided that $1-4 \lambda$ In $2>0$, i.e., $\lambda<.36067 \cdots$.

One can now see by comparing the above that Corollary 2 under the same condition on $\lambda$ gives a better information on the location of the solution than Theorem 5 [8], [9].

Our next goal is to use Theorem 4 to obtain solutions of (3.3) for a wider range of $\lambda$. It is not necessary to assume $B$ has any connection with Chandrasekhar's equation in Proposition 2 or 3.

PROPOSITION 2. If $\mathrm{z} \in \mathrm{X}$ is a solution of the equation

satisfying

$$
z=M+\lambda M_{2}(z, z),
$$

$$
2 \lambda\left\|\mathrm{M}_{2}\right\| \cdot\|z\|<1 \text {, }
$$

then for

where

$$
\lambda \leq \lambda<\mathrm{C}_{1}
$$

$$
C_{1}=\left[4\left\|M_{2}\right\|\|z\|\left(1-\lambda\left\|M_{2}\right\| \cdot\|z\|\right)\right]^{-1}
$$

the conclusions of Theorem 3 for the equation

hold.

$$
x=M_{0}+\lambda_{1} M_{2}(x, x)
$$

PROOF. To apply Thoerem 3 we need

since

$$
\|z\|<\left[2 \sqrt{\lambda_{1}\left\|\mathbb{M}_{2}\right\|}\left(\sqrt{\left|\lambda-\lambda_{1}\right| \mid \mathbb{M}_{2} \|}+\sqrt{\lambda_{1}\left\|\mathbb{M}_{2}\right\|}\right)\right]^{-1}
$$

we have

$$
\lambda_{1}<c_{1}=\left[4\left\|M_{2}\right\| \cdot\|z\|\left(1-\lambda\left\|_{2}\right\| \cdot\|z\|\right)\right]^{-1} \text {, }
$$

$$
\lambda_{1}^{2}-\lambda_{1} \lambda<\left(\frac{1}{2\left\|M_{2}\right\| \cdot\|z\|}\right)^{2}+\lambda_{1}^{2}-\frac{\lambda_{1}}{\left\|M_{2}\right\| \cdot\|z\|}
$$

or by taking the square root of both sides of the above inequality and using

we get

$$
\lambda_{1}<\left(2\left\|M_{2}\right\| \cdot\|z\|\right)^{-1}
$$

$$
\sqrt{\lambda_{1}\left(\lambda_{1}-\lambda\right)}<\frac{1}{2\left\|m_{2}\right\| \cdot\|z\|}-\lambda_{1} .
$$

The result now follows by solving the last inequality for $\|z\|$.

If $z$ is not an exact solution of the quadratic equation

$$
z=M_{0}+N_{2}(z, z),
$$

then we can use the following generalization of Proposition 2. 


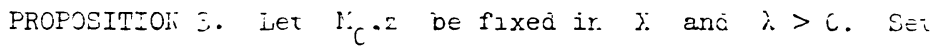

$$
\begin{aligned}
& \epsilon=\left\|\lambda v_{u_{2}}(z, z)+N_{C}-z\right\| \cdot\left\|r_{2}\right\| \cdot\|z\|^{-c}
\end{aligned}
$$

anc

$$
C_{1}=\left\{4\|z\| \mathbb{N}_{2} \|\left[I-(\lambda-\epsilon) \mathbb{M}_{2}\|\cdot\| z \|\right]\right\}^{-I} \text {. }
$$

Tnen for any $\lambda_{I}$ satisfyine $\lambda \leq \lambda_{I}<C_{I}$ the equatior

$$
x=M_{C}+\lambda_{1} N_{2}(x, x)
$$

has a unique solution in $\mathrm{U}(\mathrm{z}, \mathrm{a})$, and in fact this solution lies in $\overline{\mathrm{U}}(\mathrm{z}, \mathrm{b})$. Here

$$
\begin{gathered}
a=\frac{1}{2 \lambda_{1}\left\|M_{2}\right\|}-\|z\| \\
b=a-\left[a^{2}-\left(1-\frac{\lambda}{\lambda_{1}}\right)\|z\|^{2}-\frac{\epsilon}{\lambda_{1}}\|z\|^{2}\right]^{1 / 2} .
\end{gathered}
$$

PROOF. Similar to Proposition 2.

REMARK 3. According to Corollary 2 or Theorem 5 and the discussion following Example 1, Chandrasekhar's equation

$$
z(s)=1+\lambda M_{2}(z(s), z(s))=1+\lambda s z(s) \int_{0}^{l} \frac{z(t)}{s+t} d t
$$

has a solution $z$ provided that $\lambda<.36067376 \cdots$. But now using Proposition 3 and the iteration suggested in Remark $I$ for a suitable $x_{0}=z_{N}(\lambda)$, we can extend the range of $\lambda$ until $.424059379 \cdots$. Here are some characteristic values for $\lambda$ the norm of the corresponding approximate solution $Z_{N}(\lambda)$ and $C_{I}(\lambda)$.

\begin{tabular}{ccc}
\hline$\lambda$ & $\left\|\mathrm{z}_{\mathrm{N}}(\lambda)\right\|$ & $\mathrm{c}_{1}(\lambda)$ \\
\hline .35 & 1.44474532 & .384363732 \\
$\vdots$ & $\vdots$ & $\vdots$ \\
.38 & 1.534201867 & .394512252 \\
$\vdots$ & 1.558263525 & .399942101 \\
.4 & $\vdots$ & $\vdots$ \\
$\vdots$ & 1.59821923 & .405244331 \\
.42 & $\vdots$ & $\vdots$ \\
.423 & 1.68363661 & .420163281 \\
$\vdots$ & $\vdots$ & $\vdots$ \\
.424 & 1.69644924 & .423011429 \\
$\vdots$ & $\vdots$ & $\vdots$ \\
.424059378 & 1.70085561 & $\vdots$ \\
.424059379 & 1.700973721 & .4240590047 \\
\hline
\end{tabular}

Note that the above results coincide at least at six decimal places with the ones obtained in [2], [3] and [10].

\section{REFERENCES}

1. ARGYROS, I.K. Quadratic equations in Banach space, perturbation techniques and applications to Chandrasekhar's and related equations, Ph.D. dissertation, University of Georgia, Athens, 1984. 
2. ARGYROE. I.i., On a contractior trieorer anc appicicatione, Proc. Syme. Fure Mati.., Amer. Math. Soc. (to appear).

3. CHANDRASEKHAF, S., Radiative transfer, Dover Puti., New York, 1960.

4. DAVIS, H.I. Introduction to nonlinear differential and integral equations, Dover Publ., New York, 196:.

5. DICKSON, L.E., New first course in the theorv of equations, Join Wiley and Sons, New York, 1939 .

6. KELLEY, C.T., Solution of the Chandrasekhar h-equation by Newton's method, J. Math. Phys. 21 (7) (1980), 1625-162\&.

7. MACFARLAND, J.E., An iterative solution of the quadratic equation in Banach space, Trans. Amer. Math. Soc. (1958), 824-830.

8. RALL, L.B., Quadratic equations in Banach space, Rend. Circ. Mat. Palermo 10(1961), 314-332.

9. RALL, L.B., Solution of abstract polynomial equations by iterative methods. Mathematics Research Center, United States Army, The University of Wisconsin Technical Report 892 (1968).

10. STIBBS, D.W.N. and WEIR, R.E., On the H-functions for isotropic scattering, Monthly Not. Roy. Astron. Soc. 119(1959), 512-525. 


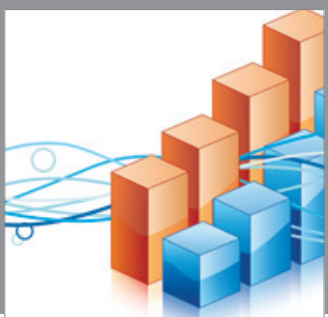

Advances in

Operations Research

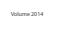

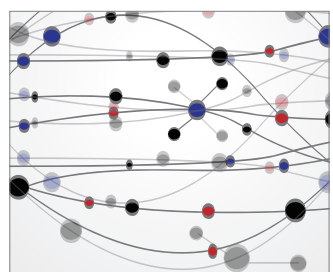

\section{The Scientific} World Journal
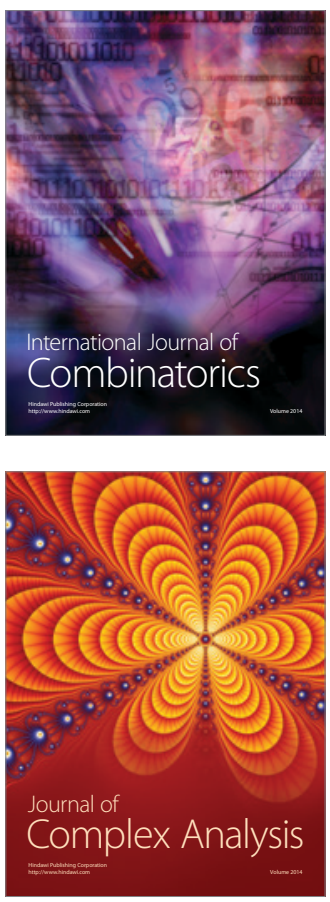

International Journal of

Mathematics and

Mathematical

Sciences
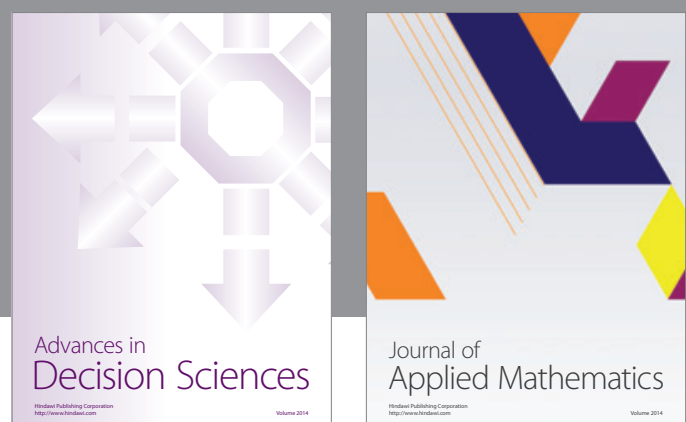

Journal of

Applied Mathematics
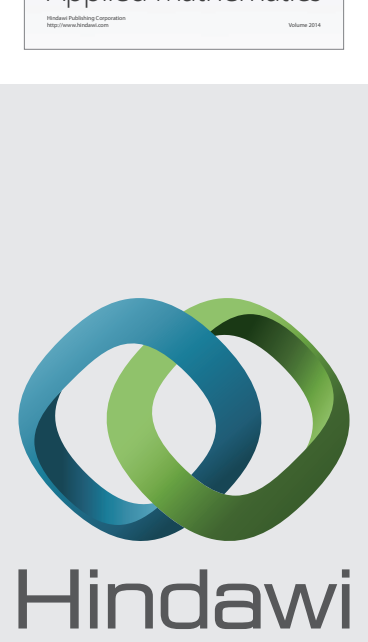

Submit your manuscripts at http://www.hindawi.com
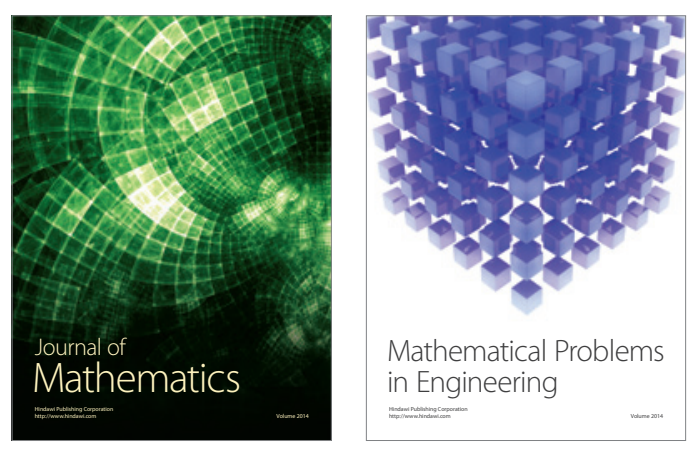

Mathematical Problems in Engineering
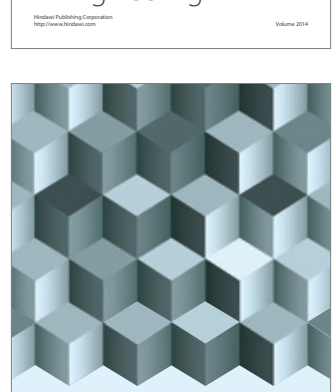

Journal of

Function Spaces
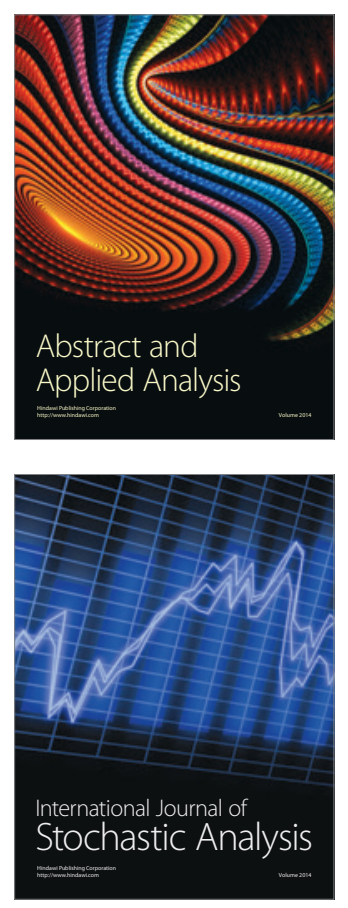

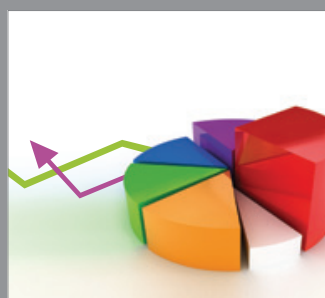

ournal of

Probability and Statistics

Promensencen
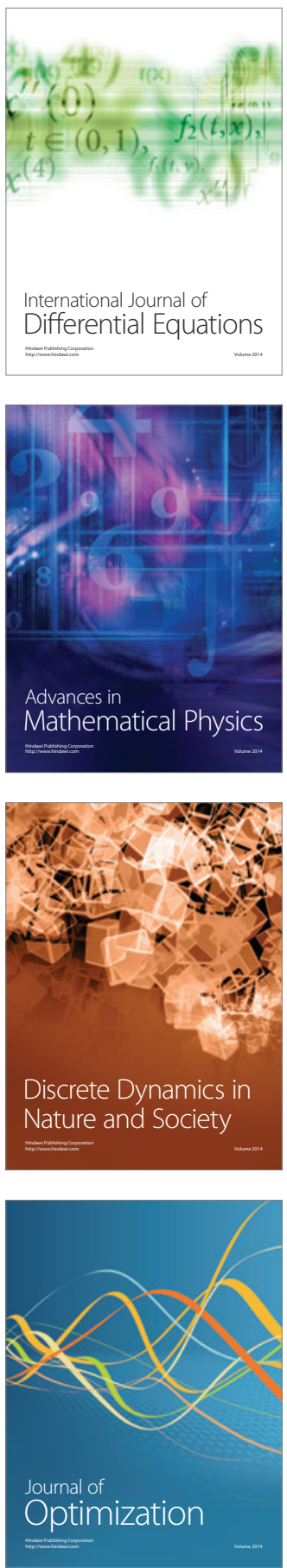